\title{
Effect of nonlinearity on dynamic diffraction and interband coupling in two-dimensional hexagonal photonic lattices
}

\author{
Bernd Terhalle, ${ }^{1,2,3}$ Anton S. Desyatnikov, ${ }^{1,}{ }^{*}$ Dragomir N. Neshev, ${ }^{1}$ Wieslaw Krolikowski, ${ }^{2}$ \\ Cornelia Denz, ${ }^{3}$ and Yuri S. Kivshar ${ }^{1}$ \\ ${ }^{1}$ Nonlinear Physics Centre, Research School of Physics and Engineering, The Australian National University, Canberra, ACT 0200, Australia \\ ${ }^{2}$ Laser Physics Centre, Research School of Physics and Engineering, The Australian National University, Canberra, ACT 0200, Australia \\ ${ }^{3}$ Institut für Angewandte Physik and Center for Nonlinear Science (CeNoS), Westfälische Wilhelms-Universität Münster,
} 48149 Münster, Germany

(Received 8 May 2012; published 16 July 2012)

\begin{abstract}
We explore experimentally the effect of nonlinearity on resonant coupling between high-symmetry momentum states in hexagonal photonic lattices. We observe nonlinear Pendellösung oscillations with the power-dependent excitation of additional critical points of the same symmetry in the reciprocal cells closest to the excitation point. In contrast, the nonlinear Landau-Zener tunneling in biased lattices exhibits a sharp transition to the modulational instability with an increase of the input optical power.
\end{abstract}

DOI: 10.1103/PhysRevA.86.013821

PACS number(s): 42.25.Bs, 42.82.Et

\section{INTRODUCTION}

The propagation of optical waves in periodic photonic structures has been attracting growing interest in recent years due to various possibilities for direct visualization of fascinating linear and nonlinear phenomena associated with wave packets and quantum particles of different natures [1,2]. Important examples in this context are resonant effects, such as Bloch oscillations [3], Landau-Zener interband tunneling [4,5], Klein tunneling [6], Zitterbewegung [7], dynamic diffraction [8], and Anderson localization in disordered systems of optical fibers [9], one-dimensional waveguide arrays [10], and twodimensional photonic lattices [11,12].

Linear Bloch oscillations and Landau-Zener interband tunneling are characterized by resonant coupling between the spectral bands which can be induced by an external force. Both phenomena have been linked recently [8] to Pendellösung oscillations, i.e., the resonant coupling of forward and Braggreflected waves in purely periodic structures without an external force [13-16]. In this case, the high-symmetry momentum states form multilevel oscillatory systems, with periodic Pendellösung transfer of population between the levels due to interference of two or more Bloch waves: a process which is similar to Rabi oscillations and energy beating in coupled waveguides. Studies of the Landau-Zener tunneling in two-dimensional optically induced hexagonal photonic latices have demonstrated that the observed dynamics is dominated by Pendellösung and Bloch oscillations without significant tunneling on a short propagation scale, whereas the tunneling process determines the asymptotic power transfer between high-symmetry points [8].

An important question in all such resonant processes is how they are affected by a possible nonlinear response of the system. In general, the nonlinearity can lead to a dramatic modification of resonant processes. For example, it may favor Anderson localization on shorter propagation distances [10] or lower levels of disorder [11]. In honeycomb lattices the Dirac dynamics breaks down $[17,18]$ under the action of

\footnotetext{
*asd124@physics.anu.edu.au
}

nonlinearity, although the Klein tunneling is not suppressed [19]. A general prerequisite for the tunneling process, such as Landau-Zener tunneling, is the coupling between spectral bands, which is usually achieved by an additional longitudinal modulation [20-22] or a linear refractive index gradient $[4,5]$. Nonlinearity provides another important mechanism to induce interband transitions via four-wave mixing (FWM). The FWM process has been shown to play an important role in the dynamics of Bose-Einstein condensates in optical lattices, where nonlinearity due to atom-atom interaction often cannot be neglected [23]. Probably the simplest arrangement in the studies of nonlinear tunneling of Bose-Einstein condensates is the double-well potential, in which the nonlinearity leads to periodic oscillations of atoms between the wells and macroscopic quantum self-trapping [24,25]. The reason for such dramatic effects is that, since the superposition principle is no longer valid in the presence of nonlinearity, the linear modes of the potential are effectively coupled.

Similarly, Bloch waves fail to represent uncoupled modes of the lattice and start to interact with each other in the presence of nonlinearity, i.e., nonlinearity effectively couples different bands and leads to significant modifications of the wave dynamics [26]. In particular, for the case of onedimensional accelerated (biased) lattices, the nonlinearity introduces asymmetry in the sense that tunneling from the ground state to the excited state is enhanced, whereas tunneling in the opposite direction becomes suppressed [27]. Nonlinear tunneling in photonic structures was studied theoretically in two-dimensional lattices, and corresponding models extending the linear Landau-Zener-Majorana systems [28-30] have been derived [31-33].

Here we study experimentally the influence of nonlinearity on the resonant coupling between high-symmetry points in two-dimensional photonic lattices. We demonstrate that in unbiased lattices, depending on the initial excitation, nonlinearity leads to tunneling of optical power to additional nearest neighbor high-symmetry points in higher Brillouin zones of the lattice. This observation suggests that systems which involve only the levels resonantly coupled in the linear limit [31-33] are insufficient to describe the nonlinear dynamics 
of resonant coupling. Furthermore, in biased lattices which exhibit Landau-Zener tunneling in the linear limit, we observe a sharp transition to modulational instability, manifesting itself in irregular output and significant broadening of the signal beam in the momentum space.

\section{EXPERIMENTAL PROCEDURE}

We consider two-dimensional hexagonal photonic lattices which are optically induced in a photorefractive crystal [34,35]. In order to compensate for the distortion of the induced refractive index pattern due to the photorefractive anisotropy, we use a stretched lattice geometry, with the horizontal and vertical lattice constants resulting in a ratio of $\eta=d_{y} / d_{x} \approx$ 2.4, compared to $\eta=\sqrt{3}$ for the unperturbed hexagonal symmetry $[8,36-38]$. A simulated intensity distribution of the lattice wave is shown in Fig. 1(a).

In our experiments, we distinguish two cases: (i) when the coupling occurs in a two-level system between the $X$ points of the Brillouin zone [Fig. 1(b)] and (ii) when it occurs in a threelevel system formed by the $M$ points as shown in Fig. 1(c). Our experimental setup is shown schematically in Fig. 2(a). A beam from a frequency-doubled Nd:YAG laser at a wavelength of $532 \mathrm{~nm}$ is sent through a combination of a half-wave plate and polarizing beam splitter in order to obtain two beams (lattice and probe beam) of controllable relative intensities. The optically induced lattice is created by a combination of Mach-Zehnder-type interferometers used to interfere three beams inside a 23-mm-long photorefractive $\mathrm{Sr}_{0.60} \mathrm{Ba}_{0.40} \mathrm{Nb}_{2} \mathrm{O}_{6}$ (SBN:Ce) crystal which is externally biased, with a dc electric field of approximately $E_{0}=2 \mathrm{kV} / \mathrm{cm}$ directed along its $c$ axis. The total power of the three interfering lattice beams is $P_{\text {latt }}=0.4 \mathrm{~mW}$ and the angles of the interfering waves are adjusted to result in lattice constants $d_{x}=24$ and $d_{y}=57 \mu \mathrm{m}$, thus giving a stretching factor of $\eta \approx 2.4$ [see Fig. 2(b)].

The probe beam is mildly focused at the front face of the crystal to a FWHM of $160 \mu \mathrm{m}$ such that it covers several lattice sites and the output is analyzed in real space as well as in Fourier space using two CCD cameras. In order to induce a refractive index gradient along the transverse $x$ direction, the crystal can be illuminated from the top with a modulated incoherent white-light intensity distribution [5]. In contrast to the theoretical analysis, in which the gradient can be oriented along any direction [30], the above technique only allows the production of a refractive index gradient along the $x$ direction. Therefore, the index gradient in our experiments (a)

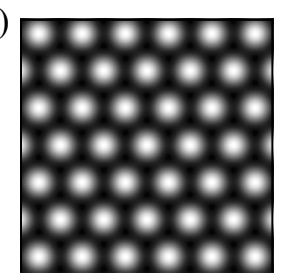

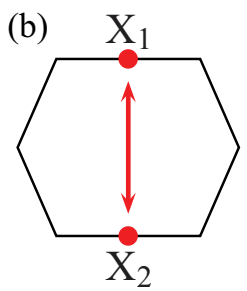

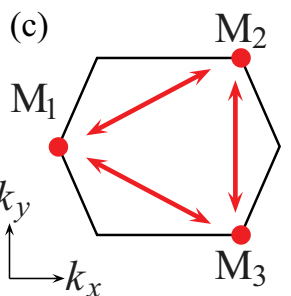

FIG. 1. (Color online) Resonant interband coupling in twodimensional hexagonal photonic lattices. (a) Simulated lattice intensity; (b) two-level system with coupling between $X$ points; (c) three-level system with coupling between $M$ points. (a)

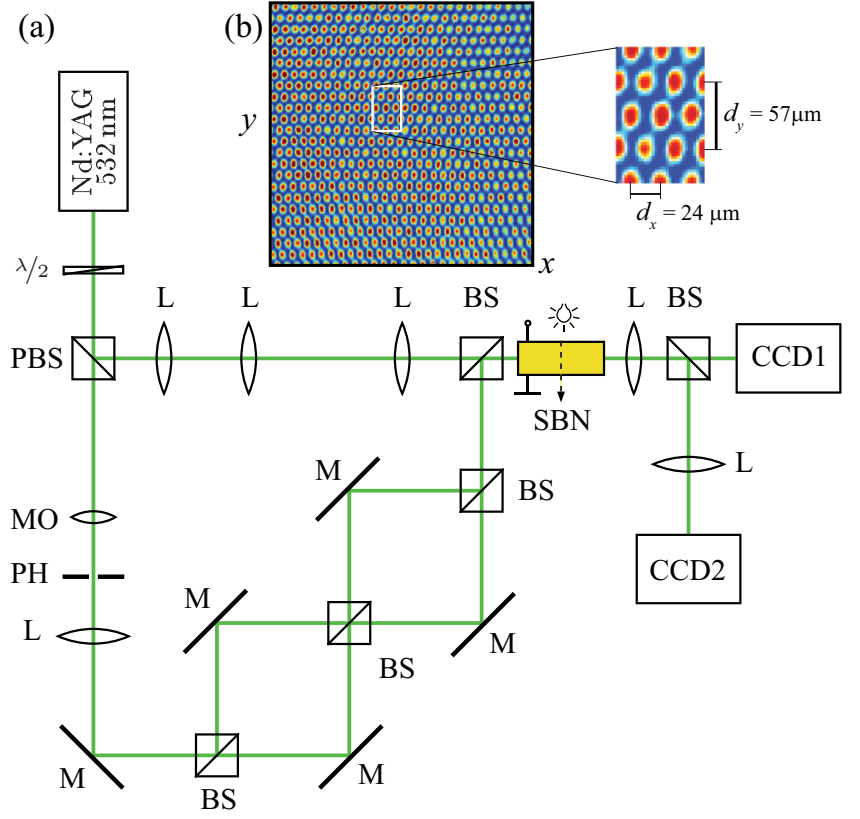

FIG. 2. (Color online) (a) Schematic experimental setup. CCD1, real-space camera; CCD2, Fourier space camera; FF, Fourier filter; L, lens; M, mirror; MO, microscope objective; (P)BS, (polarizing) beam splitter; $\mathrm{PH}$, pinhole. (b) Experimental intensity distribution of the stretched hexagonal lattice.

on Landau-Zener tunneling is always directed along the horizontal direction of the first Brillouin zone (Fig. 1).

\section{NONLINEAR PENDELLÖSUNG OSCILLATIONS}

Before analyzing the nonlinear system, we briefly recall the example of linear one-dimensional Pendellösung oscillations [8]. To this end, the input beam, with a power of $P_{\text {in }}=0.1 \mu \mathrm{W}$, is sent at such an angle of incidence as to match with the $X_{1}$ point of the Brillouin zone and the output intensity distribution is observed in Fourier space. As shown in Fig. 3, the beam gets Bragg reflected and the output contains a second peak at the $X_{2}$ point. The power ratio of the two intensity peaks changes with the applied electric field $E_{\text {ext }}=0-1 \mathrm{kV} / \mathrm{cm}$ and thus depends on the lattice depth; the latter determines the oscillation frequency [8]. It is important to note that since we are working in the linear regime, the energy is locked between the two $X$ points, with no additional points excited.
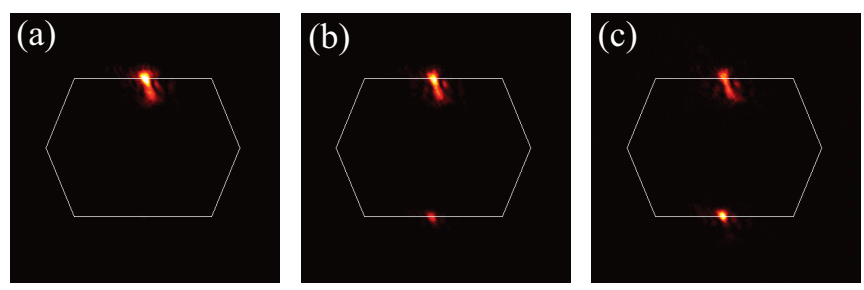

FIG. 3. (Color online) Experimentally recorded far-field intensity distributions for one-dimensional linear Pendellösung oscillations with the input beam at the top $X$ point of the Brillouin zone; the Brillouin zone is shown here and in subsequent figures by (white) hexagons. The applied field $E_{\text {ext }}$ is (a) $0.2 \mathrm{kV} / \mathrm{cm}$, (b) $0.6 \mathrm{kV} / \mathrm{cm}$, and (c) $1.0 \mathrm{kV} / \mathrm{cm}$. 
(i)
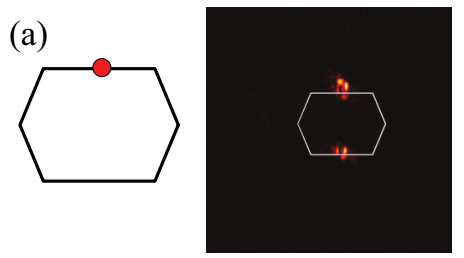

(b)

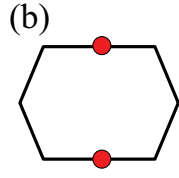

(c)

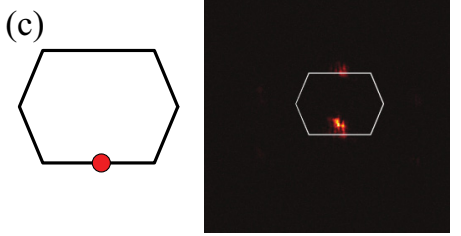

Input power $[\mu \mathrm{W}]: \quad 0.2$
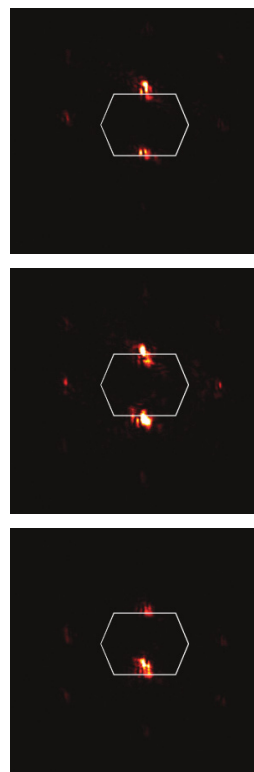

0.4
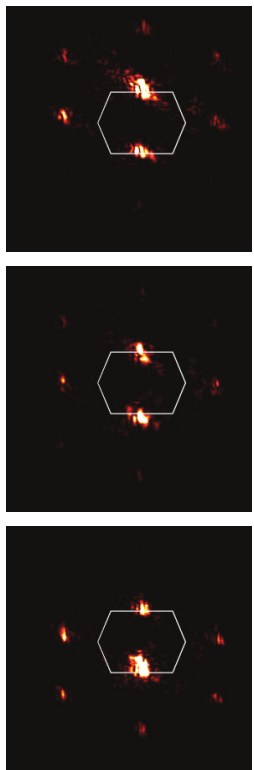

0.5
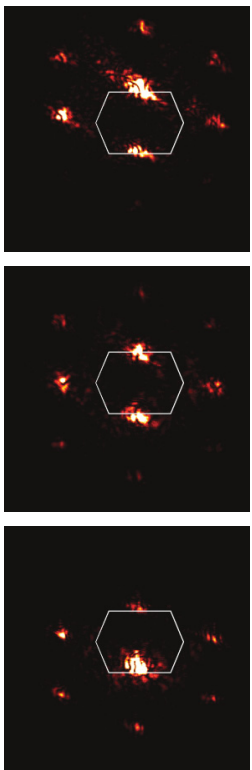

0.6
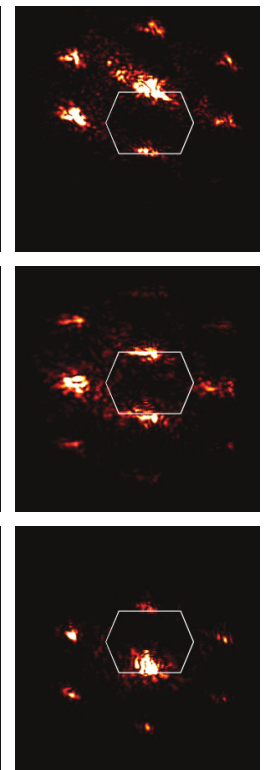

0.8
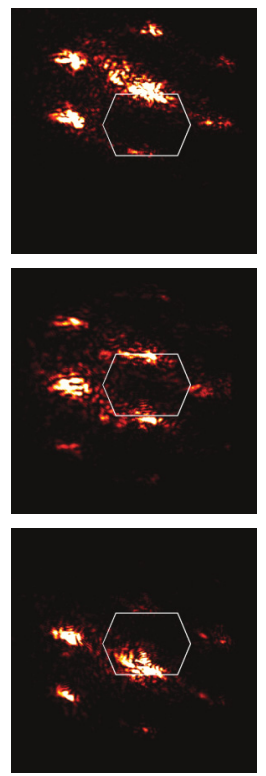

1.0

FIG. 4. (Color online) Nonlinear interband tunneling in the unbiased lattice with three excitation conditions at the $X_{1,2}$ points of the Brillouin zone, as indicated schematically in the left column: (a) input beam with momentum at the $X_{1}$ point, (b) two in-phase beam excitation, and (a) initial excitation of the $X_{2}$ point. The far-field (Fourier space) outputs are shown for different input powers, as indicated at the bottom: $P_{\text {in }}=0.2,0.4,0.5,0.6,0.8$ and $1.0 \mu \mathrm{W}$.

This situation changes when moving to the nonlinear regime, which, in our experiments in photorefractive media, is achieved by increasing the input power of the probe beam. We again adjust the input beam angle to match with the $X_{1}$ or $X_{2}$ point and observe in Figs. 4(a) and 4(c), respectively, the output intensity distributions in Fourier space with varying input powers. The externally applied electric field is $E_{\text {ext }}=$ $1 \mathrm{kV} / \mathrm{cm}$ to ensure a sufficient lattice depth. As before, the output at low power $P_{\text {in }}<0.5 \mu \mathrm{W}$ shows only two excited $X$ points. However, with an increase in power up to $P_{\text {in }}=$ $0.6 \mu \mathrm{W}$, the output changes dramatically and we observe the appearance of additional excited $X$ points in the higher Brillouin zones. A further increase in power then leads to the development of modulational instability, with significantly broadened and disordered Fourier peaks. The results presented in Figs. 4(a) and 4(c) suggest that the power is transferred to the $X$ points closest to the initially excited point in the Brillouin zone $\left(X_{1}\right.$ or $\left.X_{2}\right)$ and thus independent of the center of the Brillouin zone. Comparing Figs. 4(a) and 4(c), we see that the two additional $X$ points, on the left and on the right of the first Brillouin zone, are present in both cases. Depending on the initial conditions, three more $X$ points are excited above [Fig. 4(a)] or below [Fig. 4(c)] the first Brillouin zone.

This vertical asymmetry is eliminated in the case of twobeam excitation shown in Fig. 4(b). Here, the power is most efficiently transferred to the left and right $X$ points, while it is evenly distributed between the three top and the three bottom $X$ points, which are hardly visible. Interestingly, we observe an asymmetry in the development of modulational instability, persistent for all three excitations, namely, the left Fourier peaks get much stronger than the right ones. This appearance of modulational instability and its intrinsic relation to interband tunneling were also discussed in Ref. [39] for nonseparable square lattices.

\section{NONLINEAR LANDAU-ZENER TUNNELING}

The crucial importance of the initial conditions for the development of modulational instability was also observed in the context of asymmetry of nonlinear Landau-Zener tunneling, in agreement with our observations above. In order to fully appreciate this effect, we perform additional experiments on nonlinear Landau-Zener tunneling in the biased lattice. However, before analyzing the nonlinear tunneling process, we again recall the linear results first. We consider the simplest case of so-called symmetric tunneling [8], in which a probe beam with initial condition $k_{y}=0$ follows the applied gradient and moves across the Brillouin zone before reaching the Bragg resonance [point $\mathrm{M}_{1}$ in Fig. 1(c)]. Eventually, two additional beams appear due to resonant coupling between the Fourier amplitudes, as shown in Fig. 5.

Since in our experiment it is not possible to directly observe the evolution of the probe beam inside the crystal, we vary the incident angle of the probe beam and monitor the Fourier space output at the back face of the crystal. For a fixed crystal length
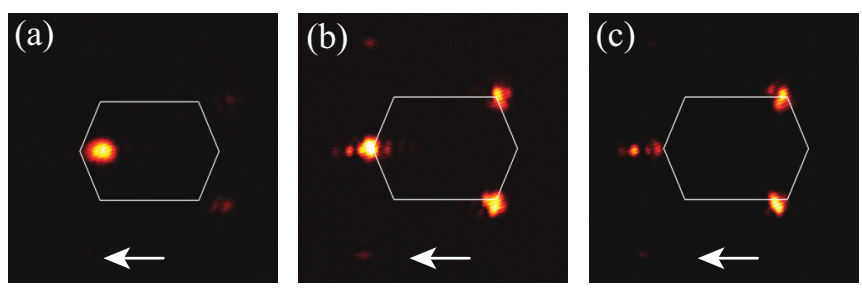

FIG. 5. (Color online) Linear Landau-Zener tunneling. Far-field output intensity distribution for different initial inclination angles $\theta$ of the probe beam: (a) $\theta=0.102^{\circ}$, (b) $\theta=0.285^{\circ}$, and (c) $\theta=0.340^{\circ}$. Arrows indicate the direction of the refractive index gradient. 

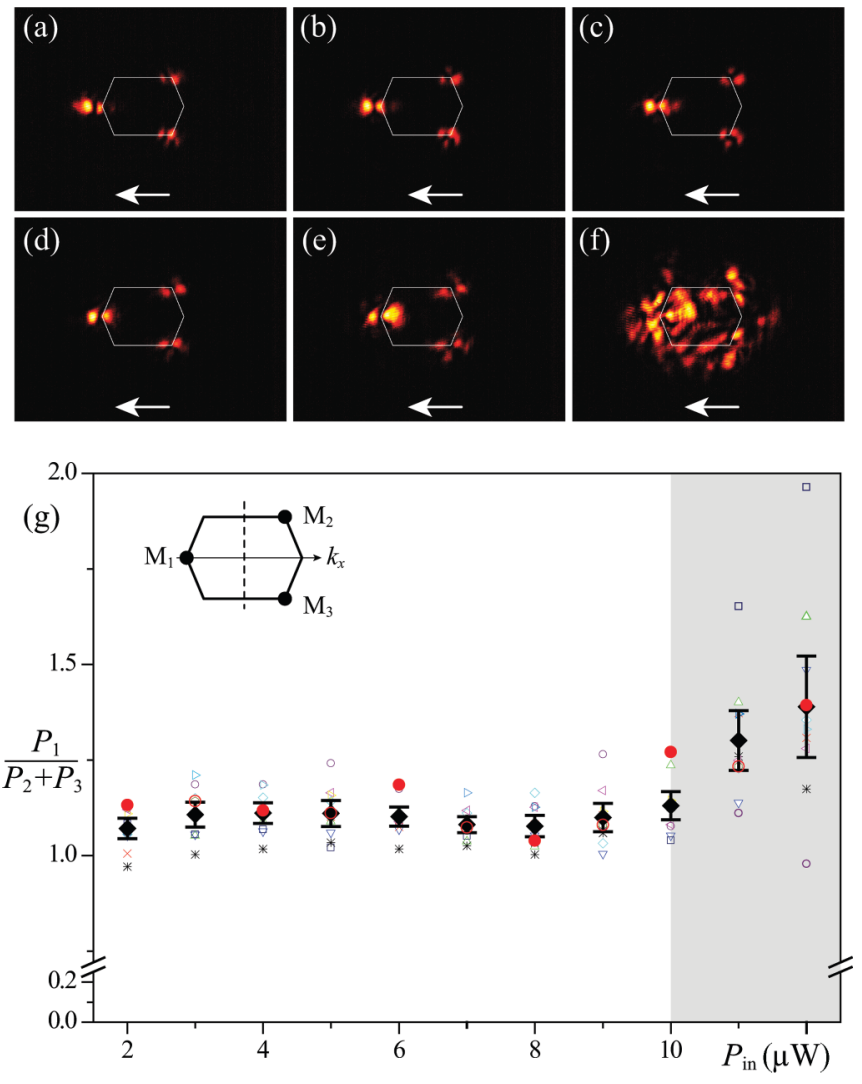

FIG. 6. (Color online) Nonlinear Landau-Zener tunneling in biased hexagonal lattices. Far-field output intensity distributions for different input powers $P_{\text {in }}$ : (a) $2 \mu \mathrm{W}$, (b) $4 \mu \mathrm{W}$, (c) $6 \mu \mathrm{W}$, (d) $8 \mu \mathrm{W}$, (e) $10 \mu \mathrm{W}$, and (f) $12 \mu \mathrm{W}$. Arrows indicate the direction of the index gradient. (g) Ratio of the output power $P_{1}$ at the initially excited $\mathrm{M}_{1}$ point to the power $P_{2}+P_{3}$ tunneled to the points $\mathrm{M}_{2}$ and $\mathrm{M}_{3}$, estimated as the ratio of intensity integrals for $k_{x}<0$ and $k_{x}>0$; see the inset. The different symbols represent 10 different measurements; filled (red) circles correspond to images (a)-(f); filled (black) diamonds and horizontal error bars show the mean value and standard deviation for 10 measurements. The area of modulational instability for $P_{\text {in }} \geqslant 10 \mu \mathrm{W}$ is shaded.

and angles below the Bragg resonance, such an excitation at different transverse wave-vector components is equivalent to different starting points in the Brillouin zone and thus allows one to infer details of the tunneling dynamics at different stages of beam evolution [5]. It was shown in [8] that the final efficiency of the tunneling process strongly depends on the initial conditions, i.e., on the initial inclination angle of the probe beam. This is also shown in Fig. 5, where the intensity of the tunneled beams in Fourier space clearly varies with the input angle.

In order to analyze the nonlinear evolution of the system, we choose a fixed angle of the input beam and vary the input power while again observing the far-field output intensity distribution, as shown in Figs. 6(a)-6(f). While nonlinear tunneling with excitation of high-symmetry points in the higher Brillouin zones was not seen, we observed a sharp transition to a modulational instability regime as shown in the Fourier image at $P_{\text {in }}=12 \mu \mathrm{W}$ [Fig. 6(f)]. To obtain a more quantitative measure of the tunneling efficiency, we integrated the powers of the three beams at the $M$ points of the first Brillouin zone. Figure 6(g) shows the obtained integrated powers of 10 repetitive measurements. The plot clearly reveals the sharp transition to the modulational instability (shaded area) which was already observed in the far-field output intensity distributions [Fig. 6(f)]. It should be noted that the left-to-right asymmetry discussed above for the case of nonlinear Pendellösung oscillations is also clearly present in this case [filled (black) diamonds in Fig. 6(g)].

\section{CONCLUSIONS}

We have experimentally studied interband coupling induced or facilitated by the nonlinear response of an optical medium and we have identified two regimes. For weak nonlinearity, the resonant tunneling in unbiased lattices, so-called Blochband tunneling [39], leads to the excitation of additional highsymmetry points in the momentum space of the lattice. For sufficiently strong nonlinearity we observe the development of modulational instability in both pure periodic and biased lattices. In addition, we observe left-to-right asymmetry of tunneling, persistent not only in biased lattices, but also in the regime of nonlinear Pendellösung oscillations.

\section{ACKNOWLEDGMENTS}

This work was supported by the Australian Research Council. B.T. acknowledges financial support from the German Academic Exchange Service (DAAD).
[1] S. Longhi, Laser Photon. Rev. 3, 243 (2009).

[2] I. Bloch, Nature Phys. 1, 23 (2005).

[3] U. Peschel, T. Pertsch, and F. Lederer, Opt. Lett. 23, 1701 (1998).

[4] H. Trompeter, T. Pertsch, F. Lederer, D. Michaelis, U. Streppel, A. Bräuer, and U. Peschel, Phys. Rev. Lett. 96, 023901 (2006).

[5] H. Trompeter, W. Krolikowski, D. N. Neshev, A. S. Desyatnikov, A. A. Sukhorukov, Yu. S. Kivshar, T. Pertsch, U. Peschel, and F. Lederer, Phys. Rev. Lett. 96, 053903 (2006).

[6] O. Bahat-Treidel, O. Peleg, M. Grobman, N. Shapira, M. Segev, and T. Pereg-Barnea, Phys. Rev. Lett. 104, 063901 (2010).
[7] F. Dreisow, M. Heinrich, R. Keil, A. Tünnermann, S. Nolte, S. Longhi, and A. Szameit, Phys. Rev. Lett. 105, 143902 (2010).

[8] B. Terhalle, A. S. Desyatnikov, D. N. Neshev, W. Krolikowski, C. Denz, and Yu. S. Kivshar, Phys. Rev. Lett. 106, 083902 (2011).

[9] T. Pertsch, U. Peschel, J. Kobelke, K. Schuster, H. Bartelt, S. Nolte, A. Tünnermann, and F. Lederer, Phys. Rev. Lett. 93, 053901 (2004).

[10] Y. Lahini, A. Avidan, F. Pozzi, M. Sorel, R. Morandotti, D. N. Christodoulides, and Y. Silberberg, Phys. Rev. Lett. 100, 013906 (2008). 
[11] T. Schwartz, G. Bartal, S. Fishman, and M. Segev, Nature 446, 52 (2007).

[12] D. M. Jović, M. R. Belić, and C. Denz, Phys. Rev. A 85, 031801(R) (2012).

[13] C. G. Shull, Phys. Rev. Lett. 21, 1585 (1968).

[14] P. J. Martin, B. G. Oldaker, A. H. Miklich, and D. E. Pritchard, Phys. Rev. Lett. 60, 515 (1988).

[15] M. L. Calvo, P. Cheben, O. Martińez-Matos, F. del Monte, and J. A. Rodrigo, Phys. Rev. Lett. 97, 084801 (2006).

[16] S. Savo, Opt. Express 16, 9097 (2008).

[17] O. Bahat-Treidel, O. Peleg, M. Segev, and H. Buljan, Phys. Rev. A 82, 013830 (2010).

[18] Z. Chen and B. Wu, Phys. Rev. Lett. 107, 065301 (2011).

[19] O. Bahat-Treidel and M. Segev, Phys. Rev. A 84, 021802 (2011).

[20] Y. V. Kartashov, V. A. Vysloukh, and L. Torner, Phys. Rev. Lett. 99, 233903 (2007).

[21] K. G. Makris, D. N. Christodoulides, O. Peleg, M. Segev, and D. Kip, Opt. Express 16, 10309 (2008).

[22] K. Shandarova, C. E. Rüter, D. Kip, K. G. Makris, D. N. Christodoulides, O. Peleg, and M. Segev, Phys. Rev. Lett. 102, 123905 (2009).

[23] O. Morsch and M. Oberthaler, Rev. Mod. Phys. 78, 179 (2006).

[24] A. Smerzi, S. Fantoni, S. Giovanazzi, and S. R. Shenoy, Phys. Rev. Lett. 79, 4950 (1997).

[25] M. Albiez, R. Gati, J. Fölling, S. Hunsmann, M. Cristiani, and M. K. Oberthaler, Phys. Rev. Lett. 95, 010402 (2005).

[26] A. Fratalocchi and G. Assanto, Phys. Rev. A 75, 013626 (2007).
[27] M. Jona-Lasinio, O. Morsch, M. Cristiani, N. Malossi, J. H. Müller, E. Courtade, M. Anderlini, and E. Arimondo, Phys. Rev. Lett. 91, 230406 (2003).

[28] V. S. Shchesnovich, S. B. Cavalcanti, J. M. Hickmann, and Yu. S. Kivshar, Phys. Rev. E 74, 056602 (2006).

[29] A. S. Desyatnikov, Yu. S. Kivshar, V. S. Shchesnovich, S. B. Cavalcanti, and J. M. Hickmann, Opt. Lett. 32, 325 (2007).

[30] V. S. Shchesnovich, A. S. Desyatnikov, and Yu. S. Kivshar, Opt. Express 16, 14076 (2008).

[31] V. S. Shchesnovich and V. V. Konotop, Phys. Rev. A 75, 063628 (2007).

[32] V. A. Brazhnyi, V. V. Konotop, V. Kuzmiak, and V. S. Shchesnovich, Phys. Rev. A 76, 023608 (2007).

[33] G.-F. Wang, D.-F. Ye, L.-B. Fu, X.-Z. Chen, and J. Liu, Phys. Rev. A 74, 033414 (2006).

[34] N. K. Efremidis, S. Sears, D. N. Christodoulides, J. W. Fleischer, and M. Segev, Phys. Rev. E 66, 046602 (2002).

[35] C. R. Rosberg, D. N. Neshev, A. A. Sukhorukov, W. Krolikowski, and Y. S. Kivshar, Opt. Lett. 32, 397 (2007).

[36] B. Terhalle, T. Richter, A. S. Desyatnikov, D. N. Neshev, W. Krolikowski, F. Kaiser, C. Denz, and Yu. S. Kivshar, Phys. Rev. Lett. 101, 013903 (2008).

[37] B. Terhalle, D. Göries, T. Richter, P. Rose, A. S. Desyatnikov, F. Kaiser, and C. Denz, Opt. Lett. 35, 604 (2010).

[38] S. Liu, Y. Hu, P. Zhang, X. Gan, C. Lou, D. Song, J. Zhao, J. Xu, and Z. Chen, Appl. Phys. Lett. 100, 061907 (2012).

[39] V. A. Brazhnyi, V. V. Konotop, and V. Kuzmiak, Phys. Rev. Lett. 96, 150402 (2006). 